\title{
Melanoma amelanótico/hipomelanótico
}

\section{Amelanotic/hypomelanotic melanoma}

\author{
Silvina Benedito ${ }^{1}$, Tomás Mosquera ${ }^{1}$, Mario Marini ${ }^{2}$, Alberto Saponaro ${ }^{3}$ y Félix Vigovich ${ }^{4}$
}

\section{RESUMEN}

El melanoma amelanótico/hipomelanótico se caracteriza por presentar, clínica y dermatoscópicamente, escaso pigmento o ninguno. Puede ocurrir en cualquiera de las variantes clínicas de melanoma. Por ser infrecuente, su diagnóstico suele retrasarse, hecho que conlleva un peor pronóstico. Se presenta como un tumor con aspecto de mácula, placa o pápula-nódulo eritematosa, con escaso pigmento 0 sin él, y mayor prevalencia en personas de más de 50 años. En la histopatología no hay hallazgos distintivos que permitan diferenciar un hipo/amelanótico de cualquier otro melanoma, por lo que el término debe reservarse solo para describir una característica clínica y dermatoscópica de un caso determinado. Su carácter de hipo/amelanótico no modifica la conducta terapéutica, que debe ajustarse a las recomendaciones de cualquier melanoma, según el estadio. Se presentan los casos de tres pacientes con esta variedad.

Palabras clave: melanoma amelanótico/hipomelanótico.

Dermatol. Argent. 2020, 26 (3): 110-113

\section{ABSTRACT}

The amelanotic/hypomelanotic melanoma is the one characterized by having a null pigment on a clinic or dermoscopic basis. It may occur in any clinic variety of melanoma. Because of its unusual characteristics, its diagnosis can take longer than expected, which entails a worse prognosis. It is found as a tumor with a taint appearance, erythematous plaque papule/nodule with scarce or null pigment, mostly found in people older than 50. In histopathology, there are no remarkable findings which allow to differentiate an hypomelanotic from any other melanoma, reason why the term should be referred to describe a clinic/ dermoscopic characteristic from a determined case. Its hypomelanotic nature does not change its therapeutic behavior, which should be adjusted to the recommendations of any other melanoma, according to the stadium. We show three patients with this variety.

Key words: amelanotic/hypomelanotic melanoma.

Dermatol. Argent. 2020, 26 (3): 110-113
1 Médico Dermatólogo de Planta, Servicio de Dermatología

2 Profesor Titular Consulto de Dermatología, Universidad de Buenos Aires

3 Jefe del Servicio de Dermatología, Docente Autorizado de la Facultad de Medicina, Universidad de Buenos Aires, Profesor Titular de Dermatología, Universidad Católica Argentina

${ }^{4}$ Médico Dermatopatólogo de Planta, Servicio de Anatomía Patológica

Hospital Británico, Ciudad Autónoma de Buenos Aires, Argentina
Contacto del autor: Silvina Benedito

E-mail: sil_be@hotmail.com

Fecha de trabajo recibido: $21 / 4 / 2020$

Fecha de trabajo aceptado: 10/8/2020

Conflicto de interés: los autores declaran que no existe conflicto de interés.

\section{INTRODUCCIÓN}

El melanoma, causante de más del $80 \%$ de las muertes por cáncer de piel, suele presentarse como un tumor pigmentado ${ }^{1}$. Las variantes clínico-patológicas clásicas son el melanoma extensivo superficial, el lentigo maligno, el melanoma nodular y el melanoma acromucolentiginoso. Cuando clínica y dermatoscópicamente no presenta pigmento, se lo denomina ame- lanótico, mientras que si presenta mínima cantidad, se lo llama hipomelanótico. Esta situación poco común, que puede darse en cualquiera de las variedades clínicas mencionadas, sobre todo en la nodular, puede ocasionar un retraso en el diagnóstico y, con ello, empeorar su pronóstico. Presentamos tres casos de esta variante clínica poco habitual. 


\section{SERIE DE CASOS}

\section{Caso clínico 1}

Una mujer de 41 años consultó por la presencia de una lesión tumoral nodular, rosada, de superficie lisa, asintomática, de $1 \mathrm{~cm}$ de diámetro, en el miembro inferior derecho, de 4 meses de evolución (Foto 1). En la dermatoscopia se observaba un borde fino hiperqueratósico, con fondo rosado y estructuras blanco-brillantes en su interior (Foto 2). Se realizó una biopsia escisional, que evidenció la presencia de un melanoma nodular en fase de crecimiento vertical, Clark III, Breslow de $1,2 \mathrm{~mm}$, no ulcerado (Foto 3). No se palpaban adenopatías. Se efectuó la ampliación de los márgenes quirúrgicos y el estudio del ganglio centinela inguinal derecho no evidenció metástasis. La tomografía computada de tórax, abdomen y pelvis no mostró indicios de enfermedad metastásica. Según la clasificación del American Joint Committee on Cancer (AJCC), correspondía a un estadio IB. Después de 6 meses desde el diagnóstico persistía sin enfermedad detectable.

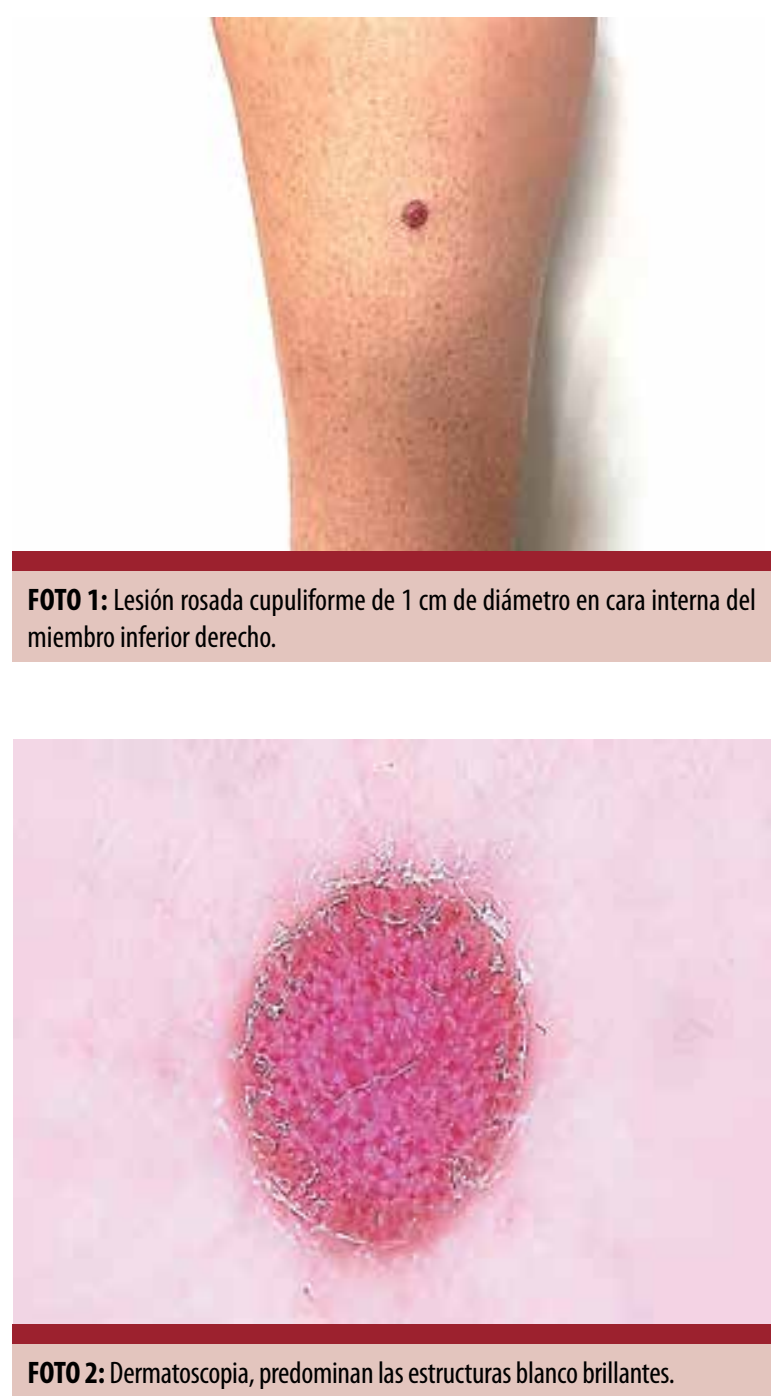

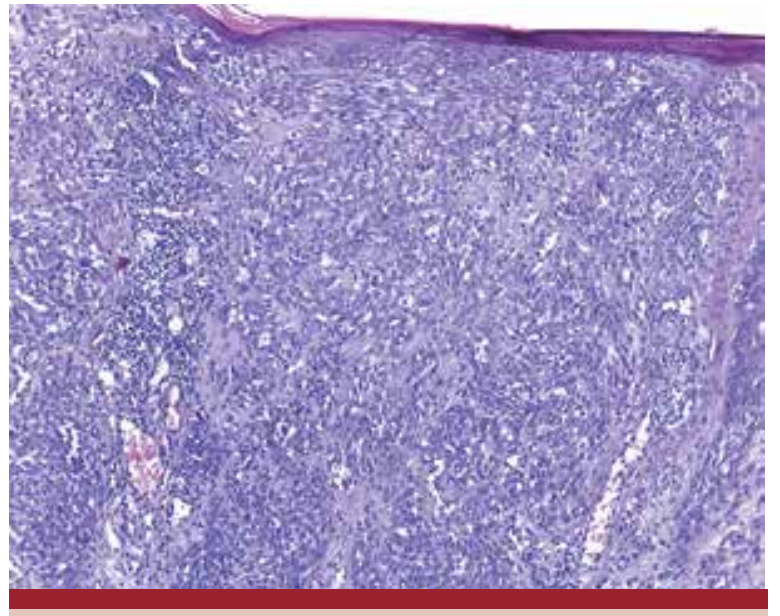

FOTO 3: Melanoma nodular en fase de crecimiento vertical, Clark III, Breslow de $1,2 \mathrm{~mm}$, no ulcerado, ausencia de pigmento (HyE, 100X).

\section{Caso clínico 2}

Un hombre de 31 años concurrió a la consulta por la aparición de una lesión tumoral de tipo nodular, eritematosa, con costra serohemática en la superficie, localizada en el área infraclavicular derecha, de 3 meses de evolución (Foto 4). En el examen dermatoscópico se constató la presencia de áreas de pigmento pequeñas, con escasos glóbulos y una costra en el centro de la lesión. El estudio histopatológico de la biopsia escisional evidenció un melanoma nodular, Clark IV, Breslow de $5 \mathrm{~mm}$, no ulcerado (Foto 5). No se palpaban adenopatías. Se realizó una PET-CT, que evidenció adenopatías hipermetabólicas en la axila derecha. Se efectuó la ampliación de los márgenes quirúrgicos y el vaciamiento ganglionar axilar derecho. La histopatología de la ampliación de los márgenes mostró la presencia de nódulos dérmicos de melanoma fusocelular y epitelioide, correspondientes a metástasis en tránsito. Se observaron metástasis de melanoma en 5 de 11 gan-

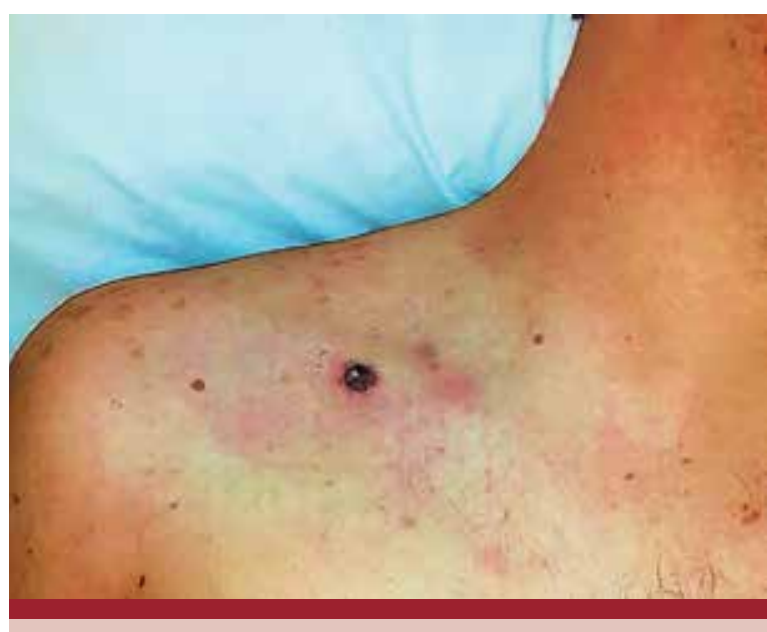

FОTO 4: Lesión nodular de 1,5 cm localizada en la región infraclavicular derecha con costra serohemática. 


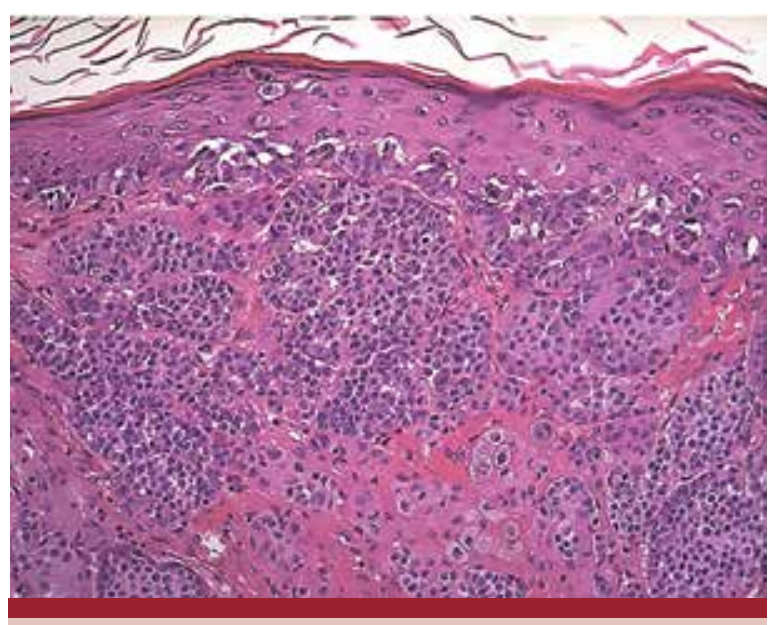

FOTO 5: Melanoma nodular, Clark IV, Breslow de $5 \mathrm{~mm}$, no ulcerado, presencia de escaso pigmento (HyE, 100X).

glios, una de ellas masiva, que se clasificó según el AJCC como estadio IV. El paciente completó el tratamiento con radioterapia del área del tumor y de la axila, e interferón alfa- $2 \mathrm{~b} 10 \mathrm{MU} / \mathrm{m}^{2}$ por vía subcutánea 5 veces por semana durante 4 semanas, seguido de $5 \mathrm{MU}$ por vía subcutánea 3 veces por semana durante 24 meses. A los 3 años desde el diagnóstico permanecía sin enfermedad.

\section{Caso clínico 3}

Un hombre de 66 años consultó por la presencia de una placa eritematocostrosa, de $3 \mathrm{~cm}$, en la región cervical posterior, de al menos un año de evolución (Foto 6). No se palpaban adenopatías. En el examen dermatoscópico se observaba una placa eritematosa con áreas blanco-brillantes, vasos puntiformes centrales y costras serohemáticas, distribuidas por casi toda la superficie. La biopsia incisional por punch evidenció un melanoma extensivo superficial, Clark III, Breslow de $0,5 \mathrm{~mm}$, ulcerado, estadio IB. Como tratamiento, se

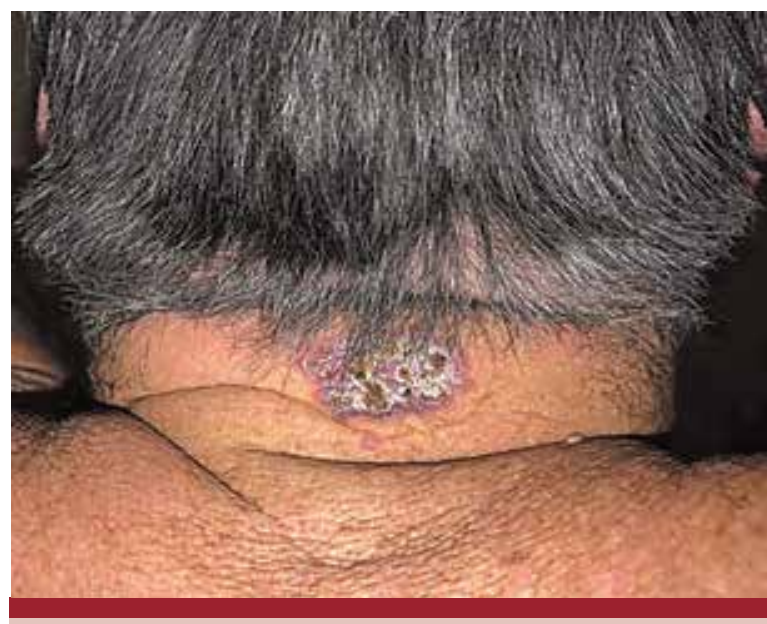

F0T0 6: Placa eritematocostrosa en la región cervical posterior de $3 \mathrm{~cm}$. realizó una extirpación simple con márgenes de $1 \mathrm{~cm}$. La histopatología de la pieza quirúrgica mostró que se trataba de un melanoma extensivo superficial en fase de crecimiento radial, con sector ulcerado, Breslow de $0,76 \mathrm{~mm}$, Clark III, sin compromiso linfovascular, perineural ni fenómenos de regresión. Después de ello, el paciente no regresó para control.

\section{COMENTARIO}

El melanoma amelanótico/hipomelanótico se caracteriza por presentar clínicamente escaso pigmento o ninguno ${ }^{2}$. Este fenómeno, que se da en el $1,8 \%$ al $8,1 \%$ de todos los casos de melanoma, puede ocurrir en cualquiera de las variantes clínicas, es más frecuente en el melanoma nodular -como se puede observar en los dos primeros casos analizados- $\mathrm{y}$, menos, en el acromucolentiginoso. Entre las variantes histopatológicas, se observa con mayor frecuencia en el desmoplásico ${ }^{3}$.

La media de edad de presentación es de 50 años, aunque es importante destacar que hasta el $50 \%$ de los casos de melanoma en la infancia son de este tipo ${ }^{4,5}$. No está claro si existen diferencias en la relación hombre/mujer. Entre los posibles factores de riesgo se mencionan el fototipo I, la etnia blanca, la ausencia de nevos en el dorso y la historia previa de melanoma amelanótico ${ }^{1}$.

Desde el punto de vista clínico -como se constata en los casos clínicos 1 y 2 - se manifiesta como un nódulo, con ulceración o no, de rápido crecimiento, o como una mácula o placa eritematosa de bordes definidos, con escaso pigmento o sin él, al igual que en el paciente del tercer caso clínico ${ }^{6,7}$. En general, no presenta los criterios del ABCD (asimetría, borde irregular, colores variados, diámetro mayor de $6 \mathrm{~mm})^{4}$. Se puede ubicar en cualquier área corporal, aunque su frecuencia varía según el sexo del paciente. En concordancia con nuestra serie de casos, en los hombres predomina en el tronco y en las mujeres, en los miembros inferiores. También hay diferencias según las distintas etnias; por ejemplo, en los asiáticos se observa más comúnmente en las zonas acrales, mientras que en los caucásicos, en las áreas de fotoexposición ${ }^{4}$.

La causa de la menor producción de melanina es poco clara. En este contexto, se cree que el melanoma amelanótico podría resultar de una insuficiente actividad de la enzima tirosinasa o de una mutación en los genes MITF (factor de transcripción asociado a microftalmía), que cumple un papel clave en la actividad de esa enzima; $M C 1 R$ (gen del receptor de la melanocortina); $p 14 A R F \mathrm{u}$ otros genes de baja penetrancia, determinantes mayores de la pigmentación de la piel y del pelo ${ }^{6,8,9}$. 
Un subgrupo de estos tumores lo constituye el melanoma 8q24, de rápido crecimiento y mal pronóstico. Tiene ganancia en el número de copias cromosómicas en $8 \mathrm{q} 24$. Esta mutación produce un aumento en la actividad del oncogén $c$-myc, que regula la función de MITF; por lo tanto, desencadena menor actividad de la enzima tirosinasa y disminuye la síntesis de melanina ${ }^{10}$.

La dermatoscopia colabora con la clínica para el diagnóstico. Las estructuras vasculares son particularmente relevantes por la escasa presencia o la total ausencia de pigmento. Se pueden observar vasos puntiformes, lineales, irregulares; lagunas; áreas rojo-lechosas $\mathrm{y}$ áreas blanco-brillantes ${ }^{11}$. Si se observa pigmento, el diagnóstico puede resultar menos dificultoso. En función de la cantidad de pigmento que puede verse con el dermatoscopio, se ha intentado clasificar el melanoma en amelanótico (carece por completo de pigmento), como en el caso de los pacientes 1 y 3 ; melanoma levemente pigmentado (menos del $25 \%$ de su superficie con pigmento), aquí se hace referencia al segundo paciente; y melanoma parcialmente pigmentado (más del $25 \%$ de la superficie de la lesión con pigmento, pero este debe ser tenue, es decir, carecer de marrón oscuro, negro o azul). Las áreas de regresión también pueden aparecer en los melanomas amelanóticos ${ }^{11}$.

El diagnóstico definitivo requiere una adecuada correlación clínico-patológica. En la histopatología no hay

\section{BIBLIOGRAFÍA}

1. Rayner JE, McMeniman EK, Duffy DL, De'Ambrosis B, et ál. Phenotypic and genotypic analysis of amelanotic and hypomelanotic melanoma patients. J Eur Acad Dermatol Venereol 2019;33:1076-1083.

2. Kuznitzky R, Garay I, Kurpis M, Ruiz Lascano A. Melanoma amelanótico. Med Cutan Iber Lat Am 2003;31:202-205.

3. Gong HZ, Zheng HY, Li J. Amelanotic melanoma. Melanoma Res 2019;29:221-230.

4. Moshe M, Levi A, Ad-El D, Ben-Amitai D, et ál. Malignant melanoma clinically mimicking pyogenic granuloma: comparison of clinical evaluation and histopathology. Melanoma Res 2018;28:363-367.

5. Ferrari A, Bono A, Baldi $M$, Collini $P$, et ál. ¿El melanoma se comporta mejor en los niños pequeños que en los adultos? Un estudio retrospectivo de 33 casos de melanoma infantil en una única institución. Pediatrics (Ed. esp.) 2005;59:147-152.

6. Zellman G. Amelanotic melanoma in a black man. J Am Acad Dermatol 1997;37:665-666.

7. García A, Ramos L, Osorio MJ, Campos Carles A. Melanoma lentigo maligno amelanótico [en línea]. Rev Argent Dermatol 2019;100(1). Disponible en: <https://rad-online.org. hallazgos distintivos -tanto con tinciones convencionales como con técnicas de inmunohistoquímica- que permitan diferenciar un hipo/amelanótico de cualquier otro melanoma, por lo que, en nuestra opinión, el término debe reservarse solo para describir una característica clínica y dermatoscópica de un caso determinado².

Entre los diagnósticos diferenciales se mencionan: granuloma piógeno, hemangioma, sarcoma de Kaposi, eccema, nevo melanocítico, queratosis seborreica, verruga vulgar, enfermedad de Bowen, queratoacantoma, carcinomas basocelular y espinocelular, poroma ecrino y algunas infecciones ${ }^{2,4,12}$.

El carácter de hipomelanótico o amelanótico puede retrasar el diagnóstico por confundirse el melanoma con otra lesión de menor relevancia. Ello se traduce en que estos melanomas, en el momento del diagnóstico, suelen presentar un espesor de Breslow elevado y están ulcerados, dos factores de mal pronóstico ${ }^{4}$.

En cuanto al tratamiento, la condición de hipo/ amelanótico no modifica la conducta terapéutica, que debe ajustarse a las recomendaciones de cualquier melanoma, según el estadio ${ }^{13}$.

Los tres casos presentados son de utilidad para retratar la importancia de una adecuada correlación clínica, dermatoscópica e histopatológica en el diagnóstico de una enfermedad grave, de características poco frecuentes.

ar/2019/03/31/melanoma-lentigo-maligno-amelanotico/> [Consulta: marzo 2020].

8. Meyle KD, Guldberg P. Genetic risk factors for melanoma. Hum Genet 2009;126:499-510.

9. Chacón M, Pfluger Y, Angel M, Waisberg F, et ál. Uncommon subtypes of malignant melanomas: a review based on clinical and molecular perspectives. Cancers 2020;2362:1-32.

10. Pouryazdanparast $P$, Brenner A, Haghighat Z, Guitart J, et ál. The role of 8 q24 copy number gains and c-MYC expression in amelanotic cutaneous melanoma. Mod Pathol 2012;25:12211226.

11. Marcucci C, Friedman P, Cesaroni E, Cohen Sabban E, et ál. Dermatoscopia del melanoma hipomelanótico y amelanótico: claves para el diagnóstico. Arch Argent Dermatol 2014;64:98-100.

12. Detrixhe A, Libon F, Mansuy M, Nikkels-Tassoudji N, et ál. Melanoma masquerading as nonmelanocytic lesions. Melanoma Res 2016;26:631-634.

13. Molgó Novell M, Reyes Baraona F, Sazunic Yáñez I. Melanoma amelanótico en una paciente con enfermedad de Parkinson. Dermatol Rev Mex 2013;57:202-205. 